\title{
REDUCTION IN THERMOELASTIC DISSIPATION IN MICROMECHANICAL RESONATORS BY DISRUPTION OF HEAT TRANSPORT
}

\author{
Rob N. Candler ${ }^{1}$, Matthew Hopcroft ${ }^{1}$, Woo-Tae Park ${ }^{1}$, Saurabh A. Chandorkar ${ }^{1}$, Gary Yama ${ }^{2}$, Kenneth E. \\ Goodson $^{1}$, Mathew Varghese ${ }^{3}$, Amy E. Duwel ${ }^{3}$, Aaron Partridge ${ }^{2}$, Markus Lutz ${ }^{2}$, and Thomas W. Kenny ${ }^{1}$ \\ ${ }^{1}$ Stanford University, Departments of Mechanical and Electrical Engineering \\ Stanford, CA 94305
}

${ }^{2}$ Robert Bosch Corporation, Research and Technology Center, North America Palo Alto, CA 94304

\section{ABSTRACT}

Micromechanical resonators have been fabricated with a novel geometry, designed to disrupt thermoelastic dissipation (TED), which is heat flow that can limit the quality factor, Q. Finite element simulations were used to design resonators with heat flow-interrupting slits that have achieved Q's higher than a simple beam achieves and higher than a simplistic application of Zener theory predicts. The new structures have a $\mathrm{Q}$ as much as $4 \mathrm{X}$ the value of those without the new geometry, 40,000 as compared to 10,000 for a $\sim 550 \mathrm{kHz}$ resonator. This result shows the necessity of modeling the complex interaction between the thermal and mechanical modes, and demonstrates a tool to engineer specific $Q$ values for micromechanical resonators.

\section{INTRODUCTION}

With the growing interest in the use of micromechanical resonators for communications applications [1], understanding the operation of these resonators has become essential. The ability to predict and control the essential parameters (e.g resonant frequency and quality factor) of these resonators is a prerequisite to their inclusion in useful systems. Quality factor, Q, determines how well the given resonator can store energy, and it has an impact on the signal-to-noise ratio of the device. There are many mechanisms that can limit the Q, including air damping, material quality, energy dissipation on the surface, anchor loss, and thermoelastic dissipation. Thermoelastic dissipation (TED) is a phenomenon, discussed in the 1930s by Zener [2], whereby a strain gradient produces a thermal gradient in a structure. The finite thermal conductivity of the material allows this thermal gradient to relax via the flow of heat across the beam. The result of this process is a loss in energy, because an energy input was required to generate the thermal gradient. This energy loss can limit the quality factor of the resonator, especially if the thermal time constant associated with the loss is of the same order as the period of the mechanical resonance. The theory developed by Zener was for simple geometries and predicted a curve of theoretically limited $\mathrm{Q}$, as shown in Fig. 1.

This theory is accurate for predicting Q limited from TED in simple beams [3]. However, the question remains open about the TED-limited Q for more complex structures. Theories have recently been developed about the effect of more complex situations, such as TED due to stress on polysilicon grains [4] and the addition of thermal paths due to process-induced geometries [5]. The present work couples novel simulation techniques [6] with a series of targeted experiments to extend TED theory and understand the interaction of multiple thermal modes with the mechanical resonance. Resonators were designed with
${ }^{3}$ Charles Stark Draper Laboratory

Cambridge, MA 02139

purposefully placed slits to disrupt the flow of heat across the flexed beams.

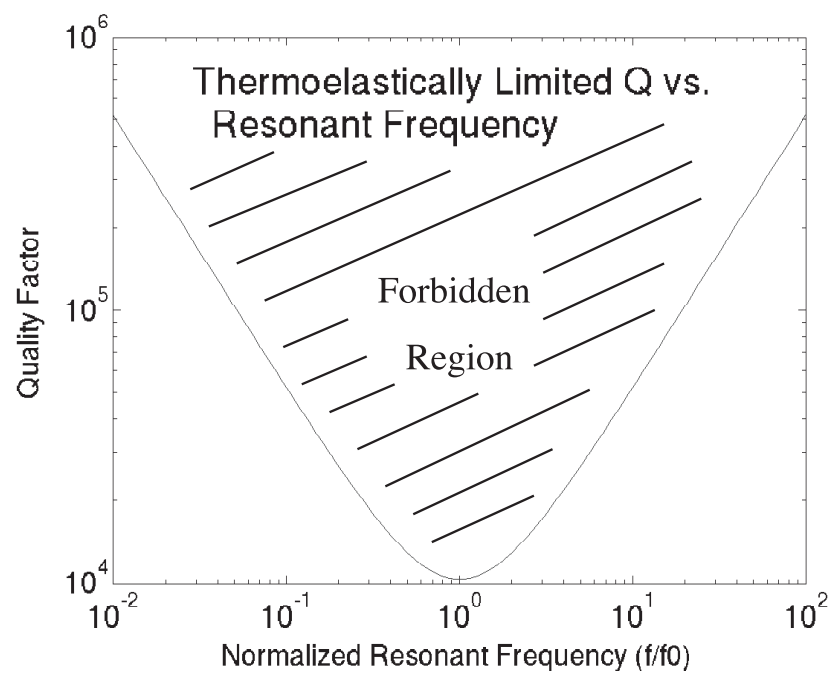

Figure 1. Theoretical limit of quality factor of a flexural beam with Zener theory.

\section{MODELING AND DESIGN}

In a weakly coupled thermoelastic model (high Q), multiple thermal modes contribute to damping, with separate weighting functions defining the damping contribution [2, 7]. In Zener's formulation, a weighting factor and a time constant for every thermal mode that contributes to damping must be calculated. For the flexural mode of a simple beam, Zener showed that a single thermal mode dominates the damping, with a weighting factor of 0.97 and a characteristic time constant determined by the beam width,

$$
\tau=\left(\frac{b}{\pi}\right)^{2} \frac{C_{v}}{\kappa}
$$

where $\tau$ is the time constant, $b$ is the width, $C_{v}$ is the specific heat per unit volume at constant pressure, and $\kappa$ is the thermal conductivity. Fig. 2 shows the Q for the single thermal mode (as

Travel support has been generously provided by the Transducers Research Foundation and by the DARPA MEMS and DARPA BioFlips programs. 
the solid line), given by the following equation using Zener's method.

$$
Q_{T E D}^{-1}=\left(\frac{E \alpha^{2} T_{0}}{C_{v}}\right) \frac{\omega \tau}{1+(\omega \tau)^{2}}
$$

$\mathrm{E}$ is the Young's Modulus, $\mathrm{T}_{0}$ is the equilibrium temperature of the beam, $\alpha$ is the coefficient of thermal expansion, and $\omega$ is the angular frequency.

The introduction of slits in the beam will modify the thermal modes of a simple beam, and multiple modes may contribute to damping. Even if one mode dominates, the weighting factor may be significantly modified. Consider the case of only one dominant mode (assumed weighting factor $=1$ ) with a characteristic time constant determined by half the beam width. The corresponding Q is plotted in Fig. 2 (as the dashed line).

Others have interpreted their data by looking for rough characteristic length scales for the thermal modes and by considering the possibility that multiple modes may contribute to $\mathrm{Q}[4,5]$. In all examples above, the weighting factors were neglected to obtain an analytically tractable solution. In general, the effect of multiple thermal modes on Q with weighting factors is as follows,

$$
Q_{T E D}^{-1}=\left(\frac{E \alpha^{2} T_{0}}{C_{v}}\right) \sum_{n} \frac{\omega \tau_{n}}{1+\left(\omega \tau_{n}\right)^{2}} f_{n}
$$

where $\tau_{n}$ is the characteristic time constant for a given mode, and $f_{n}$ is the weighting factor [7]. For arbitrary geometries, it may not be possible to identify characteristic thermal time constants by inspection. It is possible to evaluate both characteristic thermal time constants as well as weighting factors numerically, and this offers valuable intuition.

In this work, however, a very different approach is used to evaluate the $\mathrm{Q}$. We find the complex eigenvalues of the fully coupled thermo-mechanical equations using a finite elements solver $[6,8]$. The coupled thermo-mechanical equations are reformulated in $2 \mathrm{D}$ by using plane stress approximations. This reduces the number of degrees of freedom, minimizing memory requirements and reducing computation time. Fig. 3 shows the simulated eigensolutions for a simple beam and a beam with slits. The materials parameters used in the simulations are summarized in Table 1 .

\begin{tabular}{|l|l|}
\hline Silicon Parameter & Value \\
\hline Density, $\rho$ & $2330 \mathrm{~kg} / \mathrm{m}^{3}$ \\
\hline Young's Modulus, E & $157 \mathrm{GPa}$ \\
\hline Poisson ratio, $v$ & 0.3 \\
\hline Specific Heat, $\mathrm{C}_{\mathrm{v}}$ & $700 \mathrm{~J} / \mathrm{kg} \cdot \mathrm{K}$ \\
\hline Thermal Conductivity, $\kappa$ & $90 \mathrm{~W} / \mathrm{K} \cdot \mathrm{m}$ \\
\hline Coefficient thermal expansion, $\alpha$ & $2.6 \mathrm{ppm} / \mathrm{K}$ \\
\hline Ambient Temperature, $\mathrm{T}_{0}$ & $300 \mathrm{~K}$ \\
\hline
\end{tabular}

Table 1. Silicon parameters used in TED simulations

Simulated Q values as a function of frequency are also included in Fig. 2. For the beam without slits, the Zener formula with one mode matches the simulations well. When slits are included, a single $\mathrm{Q}$ minimum still appears. Interestingly, the frequency of the $\mathrm{Q}$ minimum is close to the frequency of $\mathrm{Q}$ minimum for a thermal time scale corresponding to half the beam width. The symmetry of the minimum and the value of $\mathrm{Q}$ deviate significantly from the amended Zener minimum. These simulations indicate that $\mathrm{Q}$ at a particular frequency can be improved by engineering the geometry of the resonator.

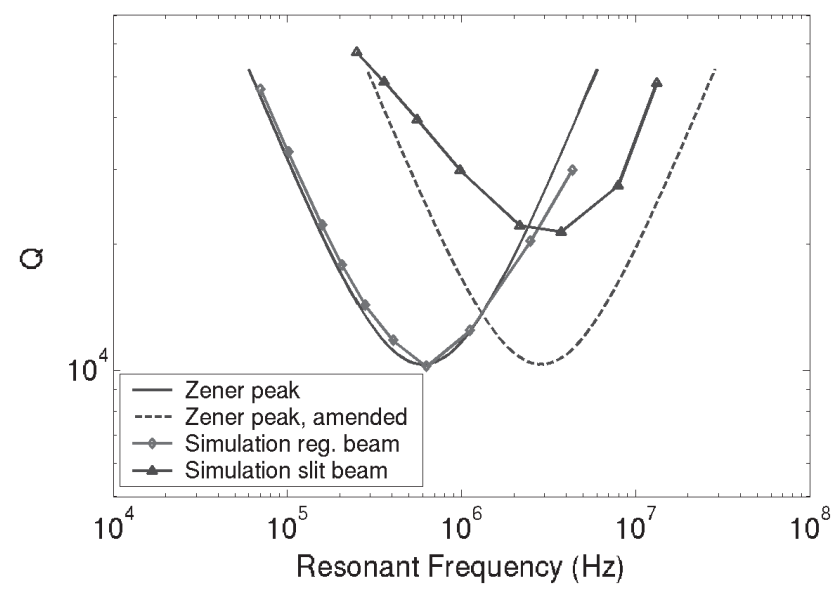

Figure 2. Theoretical limit of $Q$ by various methods: Zener theory, Zener theory amended to model a beam with slits, FEMLAB eigenvalue simulation for regular beam, and FEMLAB eigenvalue simulation for beam with slits.

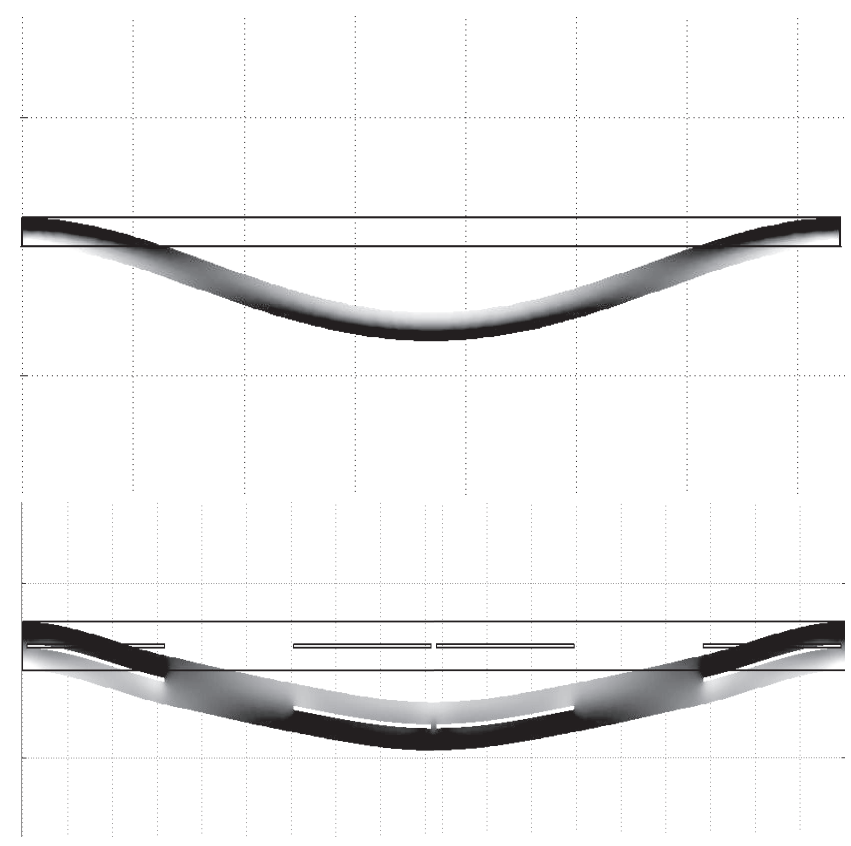

Figure 3. FEMLAB plot of eigensolutions to thermoelastic equations for a regular beam (top) and a beam with slits (bottom). Deformation shows mechanical solution, and grey scale indicates the local temperature variation. Lighter tone correlates to higher temperatures. The slits isolate the hot and cold regions and also modify the overall damping.

The resonators were designed to examine different mechanical resonant frequencies while maintaining similar thermal modes. In order to do this, a control set of resonators without slits was included in the design. The width in the direction of resonance was fixed at $12 \mu \mathrm{m}$ to keep a constant thermal time constant across the beam. The length of the beam was altered to produce resonators with different resonant frequencies. A second set of resonant beams with slits designed to disrupt the flow of heat 
across the beam was included with the purpose being to understand the effect of additional thermal paths. The structures were all doubly-clamped tuning forks, as seen in Fig. 4, with the slit configuration shown in Fig. 5.

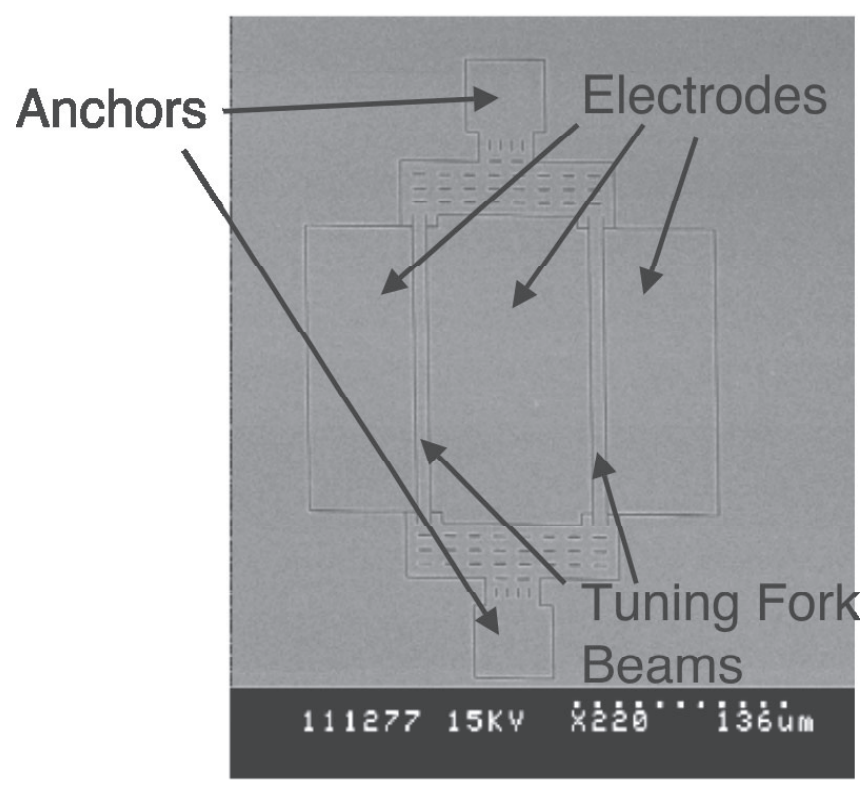

Figure 4. Top view SEM of tuning fork, showing beams, anchors, and electrodes.

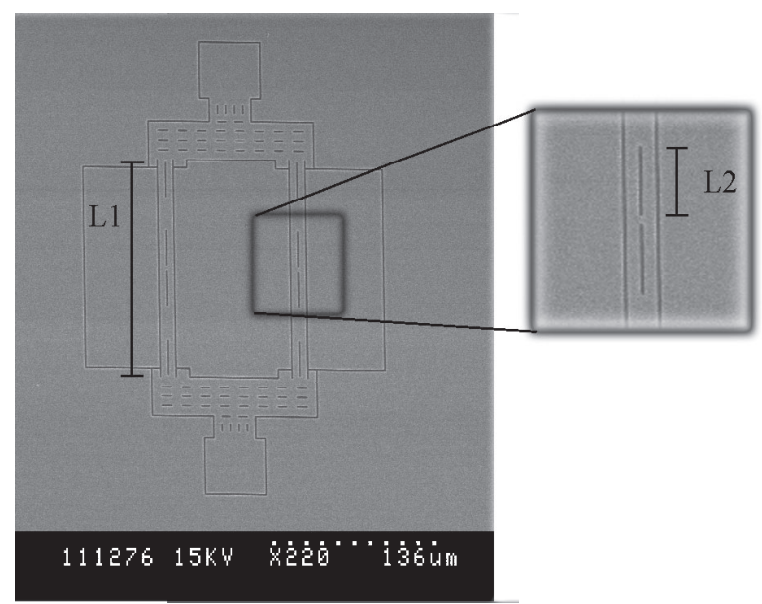

Figure 5. Top view SEM of tuning fork with heat flow interrupting slits. Each beam has 4 slits. The slit-to-beam length ratio, L2:L1, is 1:6. A range of values for $L 1$ was used to vary frequency, but the ratio of $L 2: L 1$ was kept constant.

\section{FABRICATION}

The devices were fabricated using a wafer scale, singlewafer encapsulation technique. Features of this process include a HF vapor release process and deposition of an epitaxial polysilicon encapsulation layer [9]. Process details can be seen in Figs. 6 and 7.

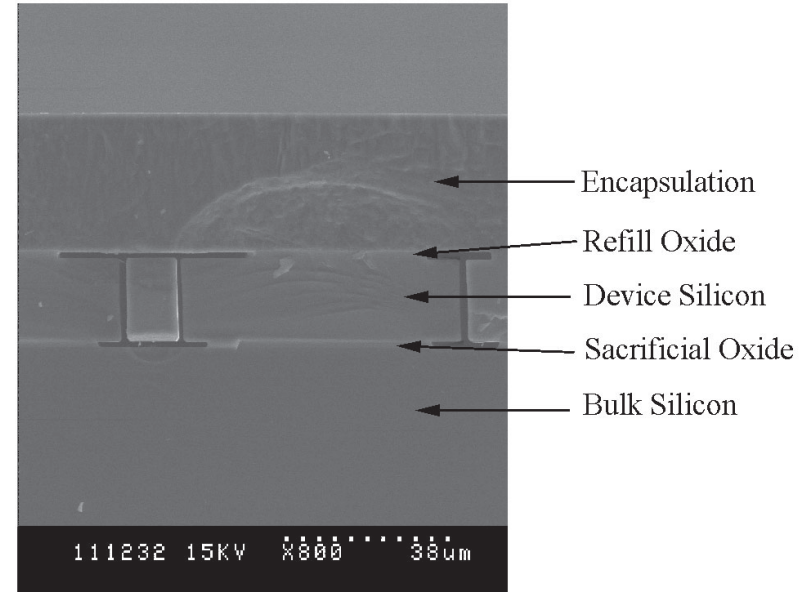

Figure 6. Cross section of fabricated wafer, showing device under silicon encapsulation.

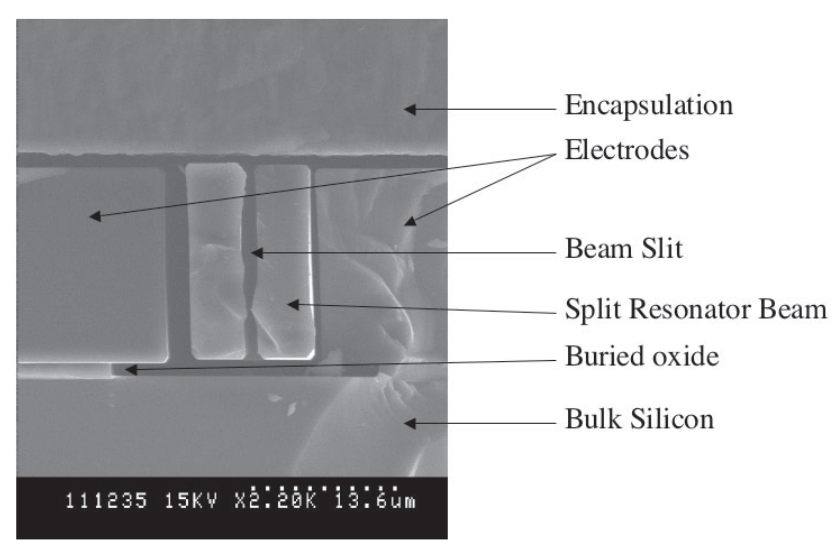

Figure 7. Cross-section of slit beam, showing electrodes on either side of a beam with a slit

\section{RESULTS}

Resonators with and without slits were fabricated and tested. A Bode Plot for a typical resonator is shown in Fig. 8.
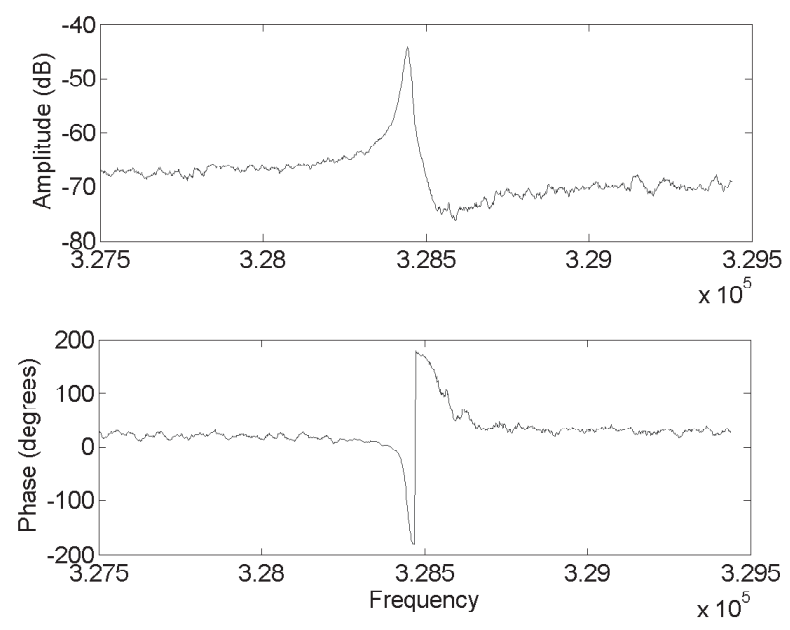

Figure 8. Bode plot for a $328.5 \mathrm{kHz}$ resonator. 
Both sets of resonators, with and without slits, were tested to determine resonant frequency and Q. Results were shown to be in good agreement with the simulations, as shown in Fig. 9.

The effect of other damping mechanisms is likely minimal with respect to the effect of TED for these measurements. The two sets of structures, with and without slits, have identical anchors, which should give them similar clamping loss. Also the addition of slits will minimally change the air damping. Since the slit beams have a similar structure but significantly higher $\mathrm{Q}$ than those without slits, it is reasonable to conclude that the beams without slits must be limited by a mechanism other than pressure or clamping, namely TED. The close following of the slit beams with simulation suggest that these results are also from TED. The parts were measured in their wafer-scale encapsulation, which was found to have a pressure of $\sim 3 \mathrm{~Pa}$. The package has shown no noticeable degradation in pressure over the past several months. In addition, the lowest frequency, highest Q parts were measured under vacuum conditions to guarantee that pressure limitation was not a factor.

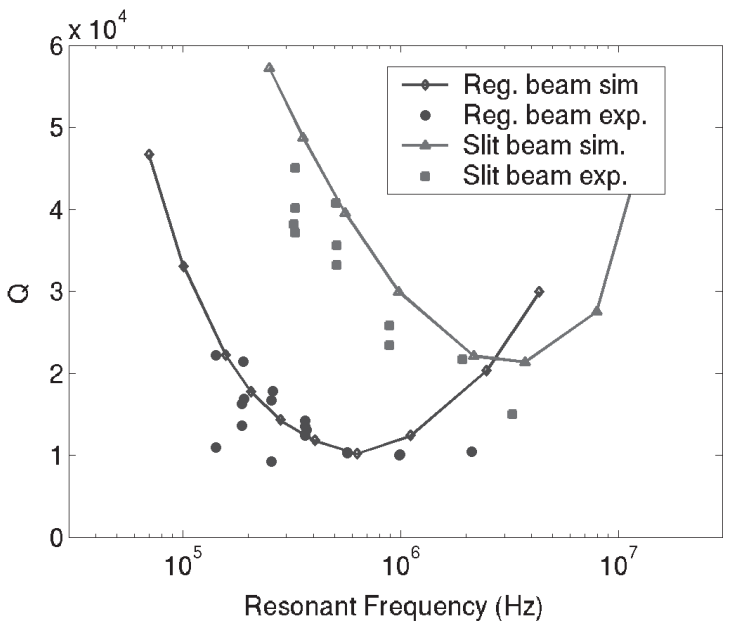

Figure 9. Simulation and experimental results for TED limited single crystal silicon resonators. Regular beams and beams with slits show different TED limitation. Both types of devices agree well with simulation results.

\section{CONCLUSION}

Novel modeling techniques have been used to study thermoelastic dissipation in complex structures and engineer resonators to improve Q. A wafer scale encapsulation process was used to fabricate devices to provide experimental evidence which, when coupled with the models, enable a more in-depth understanding of TED, and capture the complex interaction between mechanical and dissipative thermal modes. The micromechanical resonators were fabricated using the modeling as a design guide, and the experimental results were found to be in good agreement with the modeling.

\section{ACKNOWLEDGEMENTS}

This work was supported by DARPA HERMIT (ONR N66001-03-1-8942), Bosch Palo Alto Research and Technology Center, a CIS Seed Grant, The National Nanofabrication Users Network facilities funded by the National Science Foundation under award ECS-9731294, and The National Science Foundation Instrumentation for Materials Research Program (DMR 9504099).

\section{REFERENCES}

[1] C. T.-C. Nguyen, "RF MEMS for wireless applications," Device Research Conference, pp. 9-12, 2002.

[2] C. Zener, "Internal Friction In Solids: II. General Theory of Themoelastic Internal Friction," Physical Review, vol. 53, pp. 90-99, 1937.

[3] T. V. Roszhart, "Micromachined Silicon Resonators," presented at Electro International, 1991, 1991.

[4] V. T. Srikar and S. D. Senturia, "Thermoelastic damping in fine-grained polysilicon flexural beam resonators," Journal ofMicroelectromechanical Systems, vol. 11, pp. 499-504, 2002.

[5] R. Abdolvand, G. K. Ho, A. Erbil, and F. Ayazi, "Thermoelastic damping in trench-refilled polysilicon resonators," TRANSDUCERS, 12th International Conference onSolid-State Sensors, Actuators and Microsystems, vol. 1, pp. 324-327, 2003.

[6] B. Antkowiak, J. P. Gorman, M. Varghese, D. J. D. Carter, and A. Duwel, "Design of a High-Q LowImpedance, GHz-Range Piezoelectric MEMS Resonator," TRANSDUCERS, 12th International Conference on Solid-State Sensors, Actuators and Microsystems, pp. 841-846, 2003.

[7] A. S. Nowick and B. S. Berry, Anelastic Relaxation in Crystalline Solids, 1972.

[8] J. P. Gorman, "Finite Element Model of Thermoelastic Damping in MEMS, Master's Thesis," in Materials Science and Engineering: Massachusetts Institute of Technology, 2002.

[9] R. N. Candler, W.-T. Park, H. Li, G. Yama, A. Partridge, M. Lutz, and T. W. Kenny, "Single wafer encapsulation of MEMS devices," IEEE Transactions on Advanced Packaging, vol. 26, pp. 227-232, 2003. 